\title{
Erratum to development and formulation of the classification criteria for osteoarthritis
}

\author{
Cibo Huang ${ }^{1 \#}$, Zhiyi Zhang ${ }^{2 *}$, Yaolong Chen ${ }^{3,4 \#}$, Yue Zhang', Dan Xing ${ }^{5}$, Like Zhao ${ }^{1}$, Jianhao Lin ${ }^{5}$, \\ Yifang Mei ${ }^{2}$, Hsiao-Yi Lin ${ }^{6}$, Yi Zheng ${ }^{7}$, Wei-Chung Tsai ${ }^{8}$, Shengyun Liu ${ }^{9}$, Quan Jiang ${ }^{10}$, Yi Liu ${ }^{11}$, \\ Jinwei Chen ${ }^{12}$, Zhizhong Ye ${ }^{13}$, Min Chen ${ }^{1}$, Yingjuan Chen ${ }^{1}$, Cong-Qiu Chu ${ }^{14}$, Ming Gao ${ }^{1}$, Lan $\mathrm{He}^{15}$, \\ Jin Lin ${ }^{16}$, Lijun $\mathrm{Wu}^{17}$, Jianhua $\mathrm{Xu}^{18}$, Pinting Yang ${ }^{19}$, Xuewu Zhang ${ }^{20}$, Qing Jiang ${ }^{21}$, Guanghua Lei ${ }^{22}$, \\ Mengtao $\mathrm{Li}^{23}$, Wanling Yang ${ }^{24}$, Xin Gu ${ }^{25}$, Yixin $\mathrm{Zhou}^{26}$, Dongyi $\mathrm{He}^{27}$, Wei Liu ${ }^{28}$, Weiya Zhang ${ }^{29}$, \\ Changhai Ding ${ }^{30}$, Xiaofeng Zeng ${ }^{23}$; on behalf of the COACH Group
}

${ }^{1}$ Department of Rheumatology, Beijing Hospital, National Center of Gerontology, Institute of Geriatric Medicine, Chinese Academy of Medical Sciences, Beijing, China; ${ }^{2}$ Department of Rheumatology, The First Affiliated Hospital, Harbin Medical University, Harbin, China; ${ }^{3}$ EvidenceBased Medicine Center, School of Basic Medical Sciences, Lanzhou University, Lanzhou, China; ${ }^{4}$ HHO Collaborating Centre for Guideline Implementation and Knowledge Translation, Lanzhou, China; ${ }^{5}$ Department of Orthopaedics, Peking University People's Hospital, Beijing, China; ${ }^{6}$ Veterans General Hospital, Taipei and National Yang-Ming Medical University, Taipei; ${ }^{7}$ Department of Rheumatology, Beijing Chaoyang Hospital, Capital Medical University, Beijing, China; ${ }^{8}$ Department of Internal Medicine, Kaohsiung Medical College, Kaohsiung City; ${ }^{9}$ Department of Rheumatology, First Affiliated Hospital of Zhengzhou University, Zhengzhou, China; ${ }^{10}$ Department of Rheumatology and Immunology, Guang'anmen Hospital, China Academy of Chinese Medical Sciences, Beijing, China; ${ }^{11}$ Department of Rheumatology, West China Hospital, Sichuan University, Chengdu, China; ${ }^{12}$ Department of Rheumatology, Second Xiangya Hospital, Central South University, Changsha, China; ${ }^{13}$ Shenzhen Futian Hospital for Rheumatic Diseases, Shenzhen, China; ${ }^{14}$ Division of Arthritis and Rheumatic Diseases, Oregon Health \& Science University and VA Portland Health Care System, Portland, OR, USA; ${ }^{15}$ Department of Rheumatology and Immunology, the First Hospital of Xi'an Jiaotong University, Xi'an, China; ${ }^{16}$ Department of Rheumatology, The First Affiliated Hospital, Zhejiang University School of Medicine, Hangzhou, China; ${ }^{17}$ Department of Rheumatology, People's Hospital of Xinjiang Uygur Autonomous Region, Urumchi, China; ${ }^{18}$ Department of Rheumatology and Immunology, the First Affiliated Hospital of Anhui Medical University, Hefei, China; ${ }^{19}$ Department of Rheumatology and Immunology, the First Affiliated Hospital of China Medical University, Beijing, China; ${ }^{20}$ Department of Rheumatology, Peking University People's Hospital, Beijing, China; ${ }^{21}$ Department of Osteoarthrosis, Affiliated Drum Tower Hospital, Nanjing University Medical College, Nanjing, China; ${ }^{22}$ Department of Orthopedics, Xiangya Hospital, Central South University, Changsha, China; ${ }^{23}$ Department of Rheumatology, Peking Union Medical College Hospital, Chinese Academy of Medical Sciences \& Peking Union Medical College, Beijing, China; ${ }^{24}$ Department of Paediatrics and Adolescent Medicine, LKS Faculty of Medicine, The University of Hong Kong, Pokfulam, Hong Kong, China; ${ }^{25}$ Department of Rehabilitation Medicine, Beijing Hospital, National Center of Gerontology, Institute of Geriatric Medicine, Chinese Academy of Medical Sciences, Beijing, China; ${ }^{26}$ Department of Orthopedics, Beijing Jishuitan Hospital, Beijing, China; ${ }^{27}$ Department of Rheumatology and Immunology, Shanghai Guanghua Hospital, Shanghai, China; ${ }^{28}$ Department of Rheumatology and Immunology, First Teaching Hospital of Tianjin University of Traditional Chinese Medicine, Tianjin, China; ${ }^{29}$ Academic Rheumatology, University of Nottingham, Nottingham, UK; ${ }^{30}$ University of Tasmania, Tasmania, Australia

\#These authors contributed equally to this work.

Correspondence to: Weiya Zhang. Academic Rheumatology, University of Nottingham, Room A18, Academic Rheumatology Clinical Sciences Building, Nottingham City Hospital, Hucknall Road, Nottingham NG5 1PB, UK. Email: weiya.zhang@nottingham.ac.uk; Changhai Ding. Menzies Research Institute Tasmania, University of Tasmania, C. Ding, Private Bag 23, Hobart, Tasmania 7000, Australia. Email: ChangHai.Ding@utas.edu. au; Xiaofeng Zeng. Department of Rheumatology, Peking Union Medical College Hospital, Peking Union Medical College and Chinese Academy of Medical Sciences, No. 41 Damucang Hutong, Xicheng District, Beijing, China. Email: xiaofeng.zeng@cstar.org.cn.

doi: $10.21037 /$ atm-2020-29

View this article at: http://dx.doi.org/10.21037/atm-2020-29 
Erratum to: Ann Transl Med 2020;8:1068

\section{Development and formulation of the classification criteria for osteoarthritis}

In the article entitled "Development and formulation of the classification criteria for osteoarthritis" published in the Vol 8 , No 17 (September 2020) of Annals Translational Medicine (1), there are some errors in the authors' affiliation information:

(I) Yue Zhang's affiliation information should be corrected as "'Department of Rheumatology, The First Affiliated Hospital, Harbin Medical University, Harbin, China;"

(II) Dan Xing's and Jianhao Lin's affiliation information should be corrected as "5epartment of Orthopaedics, Peking University People's Hospital, Beijing, China;"

(III) Jin Lin's affiliation information should be corrected as " ${ }^{16}$ Department of Rheumatology, The First Affiliated Hospital, Zhejiang University School of Medicine, Hangzhou, China;"

(IV) Mengtao Li's and Xiaofeng Zeng's affiliation information should be corrected as “ ${ }^{23}$ Department of Rheumatology, Peking Union Medical College Hospital, Chinese Academy of Medical Sciences \& Peking Union Medical College, Beijing, China;"

(V) Wanling Yang's affiliation information should be corrected as "24 Department of Paediatrics and Adolescent Medicine, LKS Faculty of Medicine, The University of Hong Kong, Pokfulam, Hong Kong, China". Since the original affiliation information of Wanling Yang was numbered 13, the following author's affiliation information should be renumbered the same as we have now listed the authors' affiliation information in this Erratum.

(VI) Xin Gu's affiliation information should be corrected as “ ${ }^{25}$ Department of Rehabilitation Medicine, Beijing Hospital, National Center of Gerontology, Institute of Geriatric Medicine, Chinese Academy of Medical Sciences, Beijing, China;"

(VII) Wei Liu's affiliation information should be corrected as “ ${ }^{28}$ Department of Rheumatology and Immunology, First Teaching Hospital of Tianjin University of Traditional Chinese Medicine, Tianjin, China.”

The correct affiliation information as followed:

(I) Yue Zhang ${ }^{2},{ }^{2}$ Department of Rheumatology, The First Affiliated Hospital, Harbin Medical University, Harbin, China;

(II) Dan Xing ${ }^{5},{ }^{5}$ Department of Orthopaedics, Peking University People's Hospital, Beijing, China;

(III) Jianhao Lin ${ }^{5},{ }^{5}$ Department of Orthopaedics, Peking University People's Hospital, Beijing, China;

(IV) Jin Lin ${ }^{16},{ }^{16}$ Department of Rheumatology, The First Affiliated Hospital, Zhejiang University School of Medicine, Hangzhou, China;

(V) Mengtao Li $\mathrm{ir}^{23},{ }^{23}$ Department of Rheumatology, Peking Union Medical College Hospital, Chinese Academy of Medical Sciences \& Peking Union Medical College, Beijing, China;

(VI) Xiaofeng Zeng ${ }^{23},{ }^{23}$ Department of Rheumatology, Peking Union Medical College Hospital, Chinese Academy of Medical Sciences \& Peking Union Medical College, Beijing, China;

(VII) Wanling Yang ${ }^{24},{ }^{24}$ Department of Paediatrics and Adolescent Medicine, LKS Faculty of Medicine, The University of Hong Kong, Pokfulam, Hong Kong, China;

(VIII) Xin $\mathrm{Gu}^{25},{ }^{25}$ Department of Rehabilitation Medicine, Beijing Hospital, National Center of Gerontology, Institute of Geriatric Medicine, Chinese Academy of Medical Sciences, Beijing, China;

(IX) Yixin Zhou ${ }^{26},{ }^{26}$ Department of Orthopedics, Beijing Jishuitan Hospital, Beijing, China;

(X) Dongyi $\mathrm{He}^{27},{ }^{27}$ Department of Rheumatology and Immunology, Shanghai Guanghua Hospital, Shanghai, China;

(XI) Wei Liu ${ }^{28}{ }^{28}$ Department of Rheumatology and Immunology, First Teaching Hospital of Tianjin University of Traditional Chinese Medicine, Tianjin, China;

(XII) Weiya Zhang ${ }^{29},{ }^{29}$ Academic Rheumatology, University of Nottingham, Nottingham, UK;

(XIII) Changhai Ding ${ }^{30},{ }^{30}$ University of Tasmania, Tasmania, Australia.

We regret the errors. 
Open Access Statement: This is an Open Access article distributed in accordance with the Creative Commons AttributionNonCommercial-NoDerivs 4.0 International License (CC BY-NC-ND 4.0), which permits the non-commercial replication and distribution of the article with the strict proviso that no changes or edits are made and the original work is properly cited (including links to both the formal publication through the relevant DOI and the license). See: https://creativecommons.org/licenses/by-nc$\mathrm{nd} / 4.0 \%$.

\section{References}

1. Huang C, Zhang Z, Chen Y, et al. Development and formulation of the classification criteria for osteoarthritis. Ann Transl Med 2020;8:1068.

Cite this article as: Huang $\mathrm{C}$, Zhang $\mathrm{Z}$, Chen $\mathrm{Y}$, Zhang $\mathrm{Y}$, Xing D, Zhao L, Lin J, Mei Y, Lin HY, Zheng Y, Tsai WC, Liu S, Jiang Q, Liu Y, Chen J, Ye Z, Chen M, Chen Y, Chu CQ, Gao M, He L, Lin J, Wu L, Xu J, Yang P, Zhang X, Jiang Q, Lei G, Li M, Yang W, Gu X, Zhou Y, He D, Liu W, Zhang W, Ding C, Zeng X; on behalf of the COACH Group. Erratum to development and formulation of the classification criteria for osteoarthritis. Ann Transl Med 2020;8(23):1630. doi: 10.21037/atm-2020-29 